\title{
Aneuploidy of chromosome 8 and mutation of circulating tumor cells predict pathologic complete response in the treatment of locally advanced rectal cancer
}

\author{
JUE-FENG WAN $^{1,2^{*}}$, XUE-QIN LI ${ }^{3 *}$, JING ZHANG $^{1,2 *}$, LI-FENG YANG $^{1,2}$, \\ JI ZHU ${ }^{1,2}$, GUI-CHAO LI ${ }^{1,2}$, LI-PING LIANG ${ }^{1,2}$, LI-JUN SHEN ${ }^{1,2}$, HUI ZHANG ${ }^{1,2}$, \\ JING LI ${ }^{4}$, YI-TONG ZHANG ${ }^{4}$, CHANG-YUE CHEN ${ }^{4}$ and ZHEN ZHANG ${ }^{1,2}$ \\ ${ }^{1}$ Department of Radiation Oncology, Fudan University Shanghai Cancer Center; \\ ${ }^{2}$ Department of Oncology, Shanghai Medical College, Fudan University, Shanghai 200032; \\ ${ }^{3}$ Department of Oncology, Affiliated Hospital of Jining Medical University, Jining, Shandong 272000; \\ ${ }^{4}$ Department of Medical Research, Shanghai MajorMed Diagnostics Company, Shanghai 201321, P.R. China
}

Received January 24, 2018; Accepted May 22, 2018

DOI: $10.3892 / \mathrm{ol} .2018 .8831$

\begin{abstract}
Identifying patients who may or may not achieve pathologic complete response (pathCR) allows for treatment with alternative approaches in the preoperative setting. The aim of the current study was to investigate whether aneuploidy of chromosome 8 and mutations of circulating tumor cells (CTCs) could predict the response of patients with rectal cancer to preoperative chemoradiotherapy. A total of 33 patients with locally advanced rectal cancer (cT3-T4 and/or $\mathrm{cN}+$ ) treated with neoadjuvant chemoradiotherapy between September 2014 and March 2015 were recruited. Blood samples were collected from 33 patients with pre-chemoradiotherapy rectal cancer. It was demonstrated that $\geq 5$ copies of chromosome 8 was associated with pathCR (univariate logistic regression, $\mathrm{P}=0.042$ ). Of the 6 patients
\end{abstract}

Correspondence to: Professor Zhen Zhang, Department of Radiation Oncology, Fudan University Shanghai Cancer Center, 270 Dong An Road, Shanghai 200032, P.R. China

E-mail: zhenzhang6@hotmail.com

Miss Chang-Yue Chen, Department of Medical Research, Shanghai MajorMed Diagnostics Company, 3399 Kang Xing Gong Road, Building 3, Shanghai 201321, P.R. China

E-mail: changyue.chen@majorbio.com

*Contributed equally

Abbreviations: pathCR, pathologic complete response; CTCs, circulating tumor cells; CRT, preoperative chemoradiotherapy; LARC, locally advanced rectal cancer; TME, total mesorectal excision; SNVs, single nucleotide variants; LCM, laser capture microdissection; MALBAC, multiple annealing and looping-based amplification cycles

Key words: rectal cancer, circulating tumor cells, complete response, preoperative chemoradiotherapy, aneuploidy of chromosome 8 whose CTCs had $<5$ copies of chromosome 8,3 achieved pathCR $(3 / 6,50 \%)$, and of the 27 patients whose CTCs had $\geq 5$ copies of chromosome 8 obtained 3 pathCR $(3 / 27,11.1 \%$; Chi-square test, $\mathrm{P}=0.0255)$. Of the 33 patients with mutations assessed, 8 significant nonsynonymous mutations in CTCs were identified as associated with pathCR (Chi-square test, P-values range, 0.0004-0.0298; mutations in ARID1A, HDAC1, APC, ERBB3, TP53, AMER1 and AR). These results suggest that $\geq 5$ copies of chromosome 8 and 8 nonsynonymous mutations in ARID1A, HDAC1, APC, ERBB3, TP53, AMER1 AR in CTCs were associated with pathCR. This conclusion should be validated further in larger prospective studies and the long-term follow-up survival data of this study will also be reported in the future.

\section{Introduction}

Preoperative chemoradiotherapy (CRT) followed by total mesorectal excision (TME) is the standard of treatment for patients with locally advanced rectal cancer (LARC) (1-3). Preoperative CRT allows for tumor downstaging and pathologic complete response (pathCR), which is correlated with 5-year recurrence free survival (4). Therefore, identifying patients who may or may not achieve a pathCR would allow for treatment with alternative approaches in the preoperative setting $(5,6)$.

Many researchers investigate specific immunohistochemical biomarkers and genotype biomarkers as potential predictors of pathCR $(7,8)$. However, genotyping tumors may be challenging due to the invasiveness of sample collection and limited availability of tissue biopsies. Instead, circulating tumor cells (CTCs) are considered an alternative source of tumor cells present in the peripheral blood of cancer patients. The ability to isolate and profile CTCs presents an attractive option for genotyping tumors noninvasively.

In the present study, we produced an integrated workflow to investigate whether aneuploidy of chromosome 8 and mutations of CTCs can be specific predictors of pathCR to 
preoperative CRT in patients with rectal cancer. Future studies will be performed to improve the workflow and validate the conclusions in prospective and larger cohorts with long-term follow-up survival data.

\section{Materials and methods}

Patients. We recruited 33 patients with LARC (cT3-T4 and/or $\mathrm{cN}+$, The 7th edition of AJCC) treated with neoadjuvant CRT at our institution between September 2014 and March 2015. All patients signed informed consent and the Fudan University Shanghai Cancer Center Ethics Review Board approved the present study.

Patients were treated with CRT, with radiotherapy dose of $50 \mathrm{~Gy}$ and simultaneous fluorouracil-based chemotherapy. All patients received $50 \mathrm{~Gy} / 25 \mathrm{Fx}$ in the study. Surgery was generally performed 6 to 8 weeks after completion of CRT and included low anterior resection, or abdominoperineal resection using TME principles. Following surgical resection, the pathological stage of the tumor was determined according to TNM classification. Standard pathological tumor staging of the resected specimens was performed after resection in accordance with the guidelines of the College of American Pathologists, with histopathological diagnosis performed by dedicated gastrointestinal cancer pathologists. The gross tumor volume was embedded and serially sectioned for subsequent hematoxylin and eosin staining and microscopic evaluation.

Blood specimen collection and processing. Blood samples from the 33 patients with rectal cancer were obtained prior to CRT. Rectal adenocarcinoma was confirmed pathologically for all patients by biopsy. For all 33 patients, $8.5 \mathrm{ml}$ blood samples were obtained for detection of CTCs. All blood samples were collected into evacuated ACD anticoagulant tubes.

Subtraction enrichment of CTCs and identification of aneuploid CTCs. The enrichment and identification of CTCs was performed according to CTCseq ${ }^{\mathrm{TM}}$ kit instruction (Shanghai Majorbio Pharmaceutical Technology Co., Ltd., Shanghai, China). CTCs were confirmed to be negative for CD45 and either positive for PanCK staining or chromosome 8 aneuploidy. CTCs on slides were counted and imaged, and individual tumor cell coordinates were recorded to facilitate subsequent target cell identification. These slides were kept frozen at $-20^{\circ} \mathrm{C}$.

Laser capture microdissection (LCM). For LCM, samples were loaded onto the stage of a Zeiss PALM MicroBeam (Carl Zeiss AG, Oberkochen, Germany) under a 40x objective. Following microdissection with a $355-\mathrm{nm}$ laser beam, target cells were collected into AdhesiveCap 200 opaque tubes (Carl Zeiss AG). CTCs from one patient were collected in one tube; the number of CTCs in one tube varied from one to thirty. Lysis buffer ( $5 \mu \mathrm{l}$; Yikon Genomics, Shanghai, China) was then added to each sample and stored at $-20^{\circ} \mathrm{C}$.

Single-cell whole-genome amplification (WGA) with multiple annealing and looping-based amplification cycles (MALBAC). WGA of lysed cells was performed using the MALBAC method (9) following the standard protocol provided by the commercial MALBAC amplification kit (Yikon Genomics).
In summary, cells were centrifuged and collected at $8,000 \mathrm{rcf}$ for 5 min, transferred to a new $200 \mu \mathrm{l}$ PCR tube, following incubation at $50^{\circ} \mathrm{C}$ for $2 \mathrm{~h}$ and $80^{\circ} \mathrm{C}$ for $10 \mathrm{~min}$. Then, $30 \mu \mathrm{l}$ of freshly prepared preamplification mix was added to each tube and was incubated at $94^{\circ} \mathrm{C}$ for $3 \mathrm{~min}$. DNA was amplified using 8 cycles of $40 \mathrm{sec}$ at $20^{\circ} \mathrm{C}, 40 \mathrm{sec}$ at $30^{\circ} \mathrm{C}, 30 \mathrm{sec}$ at $40^{\circ} \mathrm{C}$, $30 \mathrm{sec}$ at $50^{\circ} \mathrm{C}, 30 \mathrm{sec}$ at $60^{\circ} \mathrm{C}, 4 \mathrm{~min}$ at $70^{\circ} \mathrm{C}, 20 \mathrm{sec}$ at $95^{\circ} \mathrm{C}$ and $10 \mathrm{sec}$ at $58^{\circ} \mathrm{C}$; samples were then placed on ice immediately. Amplification reaction mix (30 $\mu \mathrm{l})$ was added to each tube and incubated at $94^{\circ} \mathrm{C}$ for $30 \mathrm{sec}$ followed by 17 cycles of $20 \mathrm{sec}$ at $94^{\circ} \mathrm{C}, 30 \mathrm{sec}$ at $58^{\circ} \mathrm{C}$ and $3 \mathrm{~min}$ at $72^{\circ} \mathrm{C}$.

WGA quality control on Agilent 2100. MALBAC products were purified with 1.4x Ampure XP beads (Beckman Coulter, Inc., Brea, CA, USA) and assessed by 2100 BioAnalyzer (Agilent Technologies, Inc., Santa Clara, CA, USA). Each MALBAC product was diluted to $1 \mathrm{ng} / \mu l$. The sample volume for the analysis was $1 \mu \mathrm{l}$. A BioAnalyzer High Sensitivity DNA kit (Agilent Technologies, Inc.) was used to visualize the size range of MALBAC products. Samples were injected into the separation channel and their components were electrophoretically separated.

Target gene sequencing. A total of 36 genes (ACVR2A, SMAD4, TP53, NRAS, P65 (NFKB3), ATM, PRKCB, AR, SUMO1, PIK3CA, APC, MLH1, BRAF, HDAC1, ABL1, IGF2, TCF7L2, MLH3, MFS2, KRAS, STAT1, MYC, ERBB3, MSH2, ERBB2, TGFBR1, AMER1, PTEN, JUN, MSH3, TRIM63, CDK1, SOX9, PMS2, ARID1A and MSH6), corresponding to $105 \mathrm{~kb}$, which were frequently mutated in colorectal cancer according to previous studies $(10,11)$, were shown to be enriched. MygeneSeq technology (Morgene) was used to identify enriched genes following the manufacturer's instructions, with a $100 \mathrm{ng}$ input of MALBAC products used. In summary, barcodes were mapped to multiplex PCR products and enriched targets were identified using the MygeneSeq panel. Then, a standard shotgun library was made from amplicons using NEXTflex Rapid DNA-Seq kit Bundle with DNA Barcodes 1-24 (Bioo Scientific, Austin, TX, USA). Final concentrations of DNA samples were measured prior to sequencing using the Qubit v.2.0 fluorometer (Thermo Fisher Scientific, Inc., Waltham, MA, USA). A total of 36 genes were sequenced using 150 cycle paired-end flowcell lanes on the HiSeq4000 system (Illumina, Inc., San Diego, CA, USA). All sequence data were processed using CASAVA 1.8.1 pipeline (Illumina, Inc.) converting BCL basecall files to fastq files.

Software analysis. MALBAC primers and Illumina adaptors were trimmed by cutadapt 1.12 (12). Trimmed sequencing reads were aligned to the human genome (hg19) using the Burrows-Wheeler Aligner (BWA-0.7.12-r1039) (13). The mapping files in SAM format were converted to BAM format and sorted by SAMtools-1.2 (14). The Genome Analysis Toolkit (GATK v3.3-0-g37228af; http://www.broadinstitute.org/gatk/) was used to locally realign the sorted BAM files (15). Then, sequencing data was re-calibrated and insertion/deletion (indel)-realigned using GATK (http://www.broadinstitute. org/gatk/) before variant detection. Base-level sequencing coverage was enumerated by the Depth Of Coverage module from bedtools. 
Next, we respectively identified single nucleotide variants (SNVs) and indels from the BAM files generated with all samples using GATK UnifiedGenotyper (parameters: -mbq 20). BAM files of all samples were processed together to generate a single VCF4 file. We used the GATK VariantFiltration to filter using the parameters $\mathrm{MQ}<40$, HaplotypeScore>200.0, FS>60.0 (SNP), FS>200.0 (Indel), then the VCF file was annotated using SnpSift and snpEff and ANNOVAR. A minimum coverage depth of 10 and $>2$ reads with variant alleles was used for further filtering of SNVs. Only SNVs covered in all samples remained following filtering. SNVs in clustered regions with neighboring SNVs within $10 \mathrm{bp}$ were filtered from the data to remove false positives (16). Finally, we filtered benign SNPs from 1000g2015aug_eas and exac03nontcga, and selected the remaining variants to separate SNVs into VCF4 files.

Statistical analyses. Statistical analyses were performed using SPSS software (v.16.0, SPSS Inc., Chicago, IL, USA). Age, number of CTCs and aneuploidy were expressed as continuous variables. Categorical variables included gender, stage and presence of aneuploidy and mutations. Univariate logistic regression was used to analyze the association between clinical characteristics and aneuploidy of chromosome 8 with pathCR. The incidence of pathCR between nonsynonymous mutations and wild type genes was compared using Pearson's chi-square test.

\section{Results}

Patient and tumor characteristics. Patient and tumor characteristics are presented in Table I. All patients completed neoadjuvant treatment. Overall, 6 (18.2\%) patients had a pathCR, 21 patients got pathPR and 6 patients got pathSD. CTCs were observed in all 33 patients with a median number of 7 cells per $8.5 \mathrm{ml}$ (range 1-30). Additionally, 12 patients had 1-3 CTCs and 21 patients had >3 CTCs. Triploidy, tetraploidy, pentaploidy or $>5$ copies of chromosome 8 were observed in CTCs from rectal cancer patients (Fig. 1). There was no apparent green staining of PanCK in Fig. 1 for two reasons. First, the expression of PanCK is not necessary to identify CTCs. In our study, CTC was defined as CEP8+/DAPI+/CD45-. The high sensitivity of this method was due to the high sensitivity of CEP8. The Panck marker was only an observational biomarker in our study. Second, one possible explanation for this observation was that those PanCK negative CTCs were undergoing epithelial-mesenchymal transition (EMT). In the CTCs, triploidy, tetraploidy and $\geq 5$ copies of chromosome 8 were observed in 25, 18 and 27 patients, respectively (Table I).

Association between pathCR and chromosome 8 aneuploidy in CTCs. Aneuploidy of chromosome 8 in CTCs from 33 patients was examined. As shown in Fig. 1, triploidy, tetraploidy, pentaploidy or $>5$ copies of chromosome 8 were observed in in CTCs from rectal cancer patients. Furthermore, univariate logistic regression indicated that $\geq 5$ copies of chromosome 8 was associated with pathCR $(\mathrm{P}=0.042$; Table II). Of the patients whose CTCs had $<5$ copies of chromosome $8(n=6)$, 3 exhibited pathCR $(3 / 6,50 \%)$, and of the 27 patients whose CTCs had $\geq 5$ copies of chromosome 8,3 exhibited pathCR $(3 / 27,11.1 \%$; Chi-square test, $\mathrm{P}=0.0255)$.
Table I. Patient and tumor characteristics $(n=33)$.

Characteristics

No. of patients $(\%)$

Sex

Male

$21(63.6)$

Female

12 (36.4)

Age (years)

Mean

Median

Range

30-73

Clinical stage

II

III

$30(90.1)$

No. of CTCs

$1-3$

$12(36.4)$

$>3$

Triploidy

Yes

No

Tetraploidy

Yes

No

$\geq 5$ copies

Yes

No
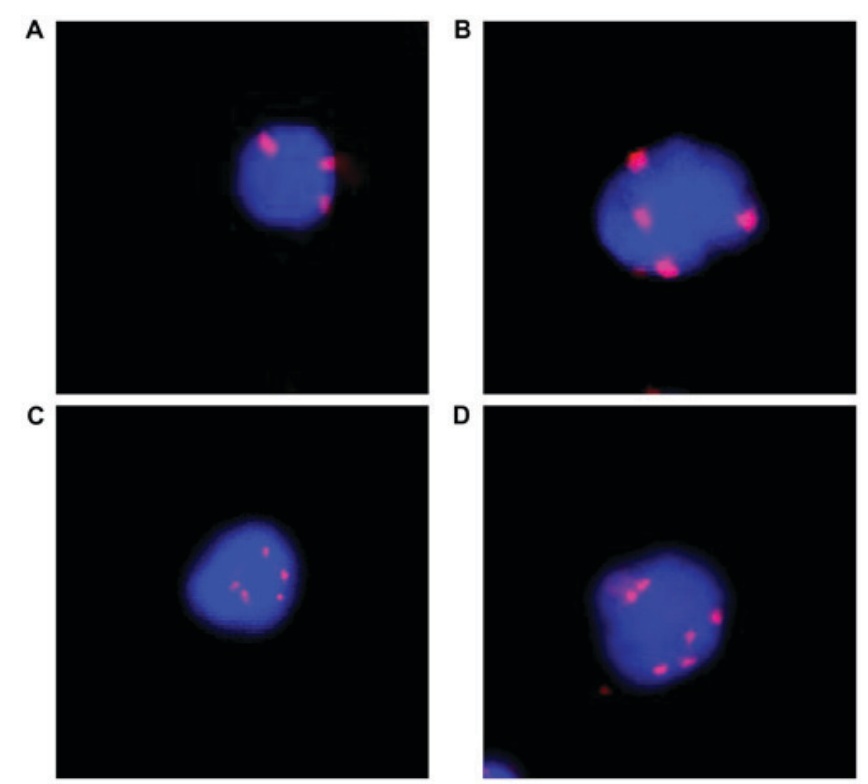

Figure 1. Images of CTCs with different chromosome 8 ploidy. (A) Triploidy, (B) tetraploidy, (C) pentaploidy and (D) $>5$ copies. Nuclei of CTCs were stained with DAPI (blue) and chromosome 8 was identified using centromere probe 8 SpectrumOrange probe (red dots).

Detection of mutational status in pathCR and non-pathCR groups. Among the 33 patients with mutations assessed, $6(18.2 \%)$ exhibited pathCR. One patient was excluded for its low coverage depth (data not shown), the average depth of the 
Table II. Univariate logistic regression predicting pathCR.

\begin{tabular}{lcc}
\hline Variables & P-value & Odds ratio \\
\hline Age & 0.16 & 1.56 \\
Sex & 0.44 & 2.63 \\
Stage & 0.34 & 2.45 \\
No. of CTCs & 0.50 & 0.94 \\
No. of triploidy & 0.56 & 0.90 \\
$\%$ triploidy & 0.10 & 10.99 \\
No. of tetraploidy & 0.30 & 0.57 \\
$\%$ tetraploidy & 0.30 & 0.03 \\
No. of $\geq 5$ copies & 0.73 & 0.97 \\
$\% \geq 5$ copies & 0.30 & 0.22 \\
Presence of triploidy & 0.64 & 1.75 \\
Presence of tetraploidy & 0.26 & 0.34 \\
Presence of $\geq 5$ copies & 0.04 & 0.13 \\
\hline
\end{tabular}

pathCR, pathologic complete response.

other 32 sequencing data was $\sim 4,000 \mathrm{x}$ (data not shown). The average coverage breadth (sites with $\geq 10 x$ coverage) varied largely from $45.5-96.18 \%$. In order to find mutational predictors of response to preoperative CRT, all 6 patients in pathCR group were analyzed, with coverage breadth (sites with $\geq 10 x$ coverage) from $50.20-73.68 \%$, and 10 patients were selected from the non-pathCR group, with coverage breadth of $\sim 80 \%$. Of the 16 patients, we assessed the mutation status of target regions that all the samples covered and selected positions with at least one SNV. We identified 22 genes that had mutations with a significant difference in frequency between the groups. These genes included TRIM63, ARID1A, HDAC1, MSH2, ACVR2A, STAT1, SUMO1, TGFBR2, APC, BRAF, ABL1, TCF7L2, IGF2, RELA, ATM, ERBB3, PRKCB, TP53, ERBB2, SOX9, AMER1 and AR. There were 64 nonsynonymous SNVs and 28 synonymous SNVs (data not shown).

Subsequently, a chi-square test was performed to examine the significant difference in pathCR group $(n=6)$ and non-pathCR group $(\mathrm{n}=10)$. Through comparing the nonsynonymous mutational status of the pathCR group and non-pathCR group, 9 statistically significant nonsynonymous mutations were identified (Chi-square test; Table IIIA).

The three most significant SNVs were TP53 p.T155I, AMER1 p.A504 V and AR p.A700 V (Chi-square, $\mathrm{P}=0.0005$ ). The results showed that p53 exon 5 had a mutation present in 5 out of $6(83.3 \%)$ pathCR patients, comparing with none of the 10 non-pathCR patients. AMER1 p.A504 V and AR p.A700 V exhibited a similar trend. P-values of the six other significant SNVs ranged from 0.0029-0.0131. The frequencies of the other gene mutations were not significantly difference between pathCR and non-pathCR patients.

Validation of mutational status in the pathCR and non-pathCR groups. To further validate the authenticity of the $9 \mathrm{SNVs}$, the SNVs were assessed in the remaining 16 patients in the non-pathCR group. There were three possible conditions for the sites analyzed: Sites with $<10 x$ coverage, wild type sites with $\geq 10 x$ coverage, mutant type sites with $\geq 10 x$ coverage. For each loci, we excluded patients with $<10 x$ coverage of the site (Table IIIB) and added the mutation status of the remaining patients into the mutations status analysis of the first 16 patients. Table IIIB shows Chi-square analysis; 8 out of 9 SNVs passed this test, and one SNV (BRAF, p.P75S) was eliminated according to the $\mathrm{P}$-value $(\mathrm{P}=0.1054)$.

The P-values changed with the addition of 16 patients. The most significant two SNVs were ERBB3 p.Y86H (Chi-square, $\mathrm{P}=0.0004)$ and AMER1 p.V490D (Chi-square, $\mathrm{P}=0.0008$ ). ERBB3 is a member of the epidermal growth factor receptor (EGFR) family of receptor tyrosine kinases genes. p.Y86H SNV in ERBB3 had a 66.7\% mutation frequency (4/6 patients) in the pathCR group, compared with a $3.8 \%$ mutation frequency (1/22 patients) in the non-pathCR group. p.V490D SNV in AMER1, a regulator of the canonical Wnt signaling pathway, had a 50\% mutation frequency in the pathCR group (3/6 patients), compared with $0 \%$ mutation frequency the in non-pathCR group (0/20 patients). The present study also showed that p.T155I in TP53 had an 83.3\% mutation frequency in the pathCR group (5/6 patients), compared with a $27.3 \%$ mutation frequency in the non-pathCR group (6/22 patients; Chi-square, $\mathrm{P}=0.0127$ ). Our study demonstrated that $8 \mathrm{SNVs}$ were significantly associated with pathCR, which is in accordance with previous reports that mutations were correlated with increased OS $(17,18)$.

\section{Discussion}

Previous reports showed the gain of chromosome 8 copies was significantly correlated with lymph node metastasis (19), and with advanced gastric cancer, pancreatic cancer and lung cancer (20-22). In the present study, we focused on whether aneuploidy of chromosome 8 and gene mutations of CTCs could assist in predicting the response to preoperative CRT in rectal adenocarcinoma patients. Overall, 18.2\% (6 out of 33) of patients achieved a pathCR following CRT. Notably, $\geq 5$ copies of chromosome 8 was associated with pathCR by univariate logistic regression ( $\mathrm{P}=0.042$, Table II), which is in accordance with Chen et al (23). Chen reported that patients who achieved a pathCR had significantly fewer high copy gains overall than non-pathCR patients $(\mathrm{P}=0.01)(23)$.

To investigate the influence of gene mutations of CTCs on response to preoperative chemoradiation, we developed a workflow to genotype of CTCs from patients. In summary, we captured CTCs, amplified the DNA and sequenced. The coverage breadth (sites with $\geq 10 x$ coverage) in pathCR group ranged from 50.20-73.68\%. For the issues of coverage breadth and the limited number of pathCR patients, all six patients in pathCR group and ten patients in non-pathCR group with coverage breadth (sites with $\geq 10 x$ coverage) $>80 \%$ were used in initial analysis. Among all the covered regions, 64 nonsynonymous SNVs were identified in the 16 patients. Of the 64 nonsynonymous SNVs, the frequencies of 9 nonsyonymous SNVs (Table IIIA) were significantly different between the pathCR group and non-pathCR group. The 9 SNVs were subsequently analyzed in the remaining 16 patients in the non-pathCR group and added the mutation status of these patients to that of the first 16 patients; following this, 8 SNVs exhibited significant differences between 
Table III. Mutations with significant difference in frequency between pathCR and non-pathCR patients.

A, Mutations between pathCR $(n=6)$ and non-pathCR patients $(n=10)$

\begin{tabular}{|c|c|c|c|c|c|c|}
\hline Gene & Amino acid change & $\begin{array}{c}\text { Overall }(\%) \\
n=16\end{array}$ & pathCR $(\%) \mathrm{n}=6$ & $\begin{array}{l}\text { Non-pathCR } \\
\quad(\%) \mathrm{n}=10\end{array}$ & Excluded & P-value \\
\hline ARID1A & p.L2117M & $3(18.8)$ & $3(50)$ & $0(0)$ & - & 0.0131 \\
\hline HDAC1 & p.F341V & $4(25)$ & $4(66.7)$ & $0(0)$ & - & 0.0029 \\
\hline APC & p.K1310M & $4(25)$ & $4(66.7)$ & $0(0)$ & - & 0.0029 \\
\hline BRAF & p.P75S & $4(25)$ & $4(66.7)$ & $0(0)$ & - & 0.0029 \\
\hline ERBB3 & p.Y86H & $4(25)$ & $4(66.7)$ & $0(0)$ & - & 0.0029 \\
\hline TP53 & p.T155I & $5(31.3)$ & $5(83.3)$ & $0(0)$ & - & 0.0005 \\
\hline AMER1 & p.A504V & $5(31.3)$ & $5(83.3)$ & $0(0)$ & - & 0.0005 \\
\hline AMER1 & p.V490D & $3(18.8)$ & $3(50)$ & $0(0)$ & - & 0.0131 \\
\hline AR & p.A700V & $5(31.3)$ & $5(83.3)$ & $0(0)$ & - & 0.0005 \\
\hline
\end{tabular}

B, Mutations between pathCR $(n=6)$ and non-pathCR patients (total number=16-26, some patients were excluded)

\begin{tabular}{|c|c|c|c|c|c|c|}
\hline Gene & Amino acid change & Overall (\%) & pathCR $(\%) \mathrm{n}=6$ & Non-pathCR (\%) & Excluded & P-value \\
\hline ARID1A & p.L2117M & $5(15.6)$ & $3(50)$ & $2(7.7)$ & 0 & 0.0101 \\
\hline HDAC1 & p.F341V & $8(30.8)$ & $4(66.7)$ & $4(20)$ & 6 & 0.0298 \\
\hline APC & p.K1310M & $9(29.0)$ & $4(66.7)$ & $5(20)$ & 1 & 0.0237 \\
\hline BRAF & p.P75S & $10(38.5)$ & $4(66.7)$ & $6(30)$ & 6 & 0.1054 \\
\hline ERBB3 & p.Y86H & $5(17.9)$ & $4(66.7)$ & $1(4.5)$ & 4 & 0.0004 \\
\hline TP53 & p.T155I & $11(39.3)$ & $5(83.3)$ & $6(27.3)$ & 4 & 0.0127 \\
\hline AMER1 & p.A504V & $12(44.4)$ & $5(83.3)$ & $7(33.3)$ & 5 & 0.0297 \\
\hline AMER1 & p.V490D & $3(11.5)$ & $3(50)$ & $0(0)$ & 6 & 0.0008 \\
\hline $\mathrm{AR}$ & p.A700V & $9(40.9)$ & $5(83.3)$ & $4(25)$ & 10 & 0.0132 \\
\hline
\end{tabular}

pathCR, pathologic complete response.

the groups (Table IIIB). For example, p.T155I in TP53 was detected in $83.3 \%$ of patients in the pathCR group, compared with $27.3 \%$ of the non-pathCR group (6/22 patient; Chi-square, $\mathrm{P}=0.0127$ ). Although the impact of $\mathrm{p} 53$ mutations on patient survival remains unclear, previous work has showed that the Arg variant of codon 72 , located in exon 4 of p53 gene, enhances the pro-apoptotic ability of the protein $(24,25)$. Patients in the present study may have a similar activating mutation in $\mathrm{p} 53$.

The other 7 SNVs had mutation frequencies of 50-83.3\% in pathCR group compared with $0-33.3 \%$ in the non-pathCR group (Chi-square, $\mathrm{P} \leq 0.0131$ ). The present study showed that mutations in 8 SNVs were significantly associated with pathCR, which in in accordance with previous reports that various mutations are correlated with increased OS $(17,18)$.

Our current workflow has several limitations that should be overcome in subsequent studies, including the WGA success rate. Our results demonstrated that the WGA success rate $[\geq 80 \%$ coverage breadth (sites with $\geq 10 x$ coverage)] of LCM-dissected CTC samples was 30.3\% (10 out of 33 CTCs) in 36-gene study; however, when the MALBAC technique was used previously on live single cells, and the WGA success rate was $\sim 90 \%$ (18) (data not shown). The low WGA amplification was possibly due to the poor DNA quality caused by the workflow. However, the current CTCs method has high sensitivity compared with other variable CTC methods and we are still improving the protocol to reduce damage to CTCs and increase the WGA success rate (26). Improvements in the following aspects may improve the WGA amplification: Optimizing the fixation conditions to reduce the degradation of DNA; and extending the lysis time to uncover the DNA template.

In conclusion, the present study suggests that the number of chromosome 8 copies and 8 nonsynonymous mutations (in ARID1A, HDAC1, APC, ERBB3, TP53, AMER1and AR) in CTCs were associated with pathCR. Out of 6 patients whose CTCs had $<5$ copies of chromosome 8,3 had pathCR $(3 / 6$ patient, $50 \%$ ) and of 27 patients whose CTCs had $\geq 5$ copies of chromosome 8 only 3 had pathCR (3/27 patients, $11.1 \%$; Chi-square test, $\mathrm{P}=0.0255)$. The $8 \mathrm{SNVs}$ were reported had mutation frequencies of $50-83.3 \%$ in the pathCR group compared with 0-33.3\% mutation frequency in the non-pathCR group. In the future we aim to perform larger, prospective studies and analyze long-term follow-up survival data.

\section{Acknowledgements}

Not applicable. 


\section{Funding}

The present study was funded by National Natural Science Foundation of China (grant no. 81372432).

\section{Availability of data and materials}

The datasets used and analyzed during the current study are available from the corresponding author on reasonable request.

\section{Authors' contributions}

JFW, XQL, JZ and ZZ conceived of and designed the study. JFW, XQL, JZ, CYC, LFY, JZ, GCL, LPL, LJS, HZ, JL and YTZ performed the analyses. JFW, XQL, JZ, CYC, LFY, JZ, GCL, LJS and $\mathrm{HZ}$ prepared all the tables. JFW, CYC, JZ and XQL wrote the main manuscript. All authors reviewed the manuscript.

\section{Ethics approval and consent to participate}

All procedures performed in studies involving human participants were in accordance with the ethical standards of the institutional and/or national research committee and with the 1964 Helsinki declaration and its later amendments or comparable ethical standards. All patients signed informed consent and the Fudan University Shanghai Cancer Center Ethics Review Board approved the study.

\section{Consent for publication}

Informed consent was obtained from all individual participants included in the present study.

\section{Competing interests}

The authors declare no competing interests.

\section{References}

1. Aschele C, Cionini L, Lonardi S, Pinto C, Cordio S, Rosati G, Artale S, Tagliagambe A, Ambrosini G, Rosetti P, et al: Primary tumor response to preoperative chemoradiation with or without oxaliplatin in locally advanced rectal cancer: Pathologic results of the STAR-01 randomized phase III trial. J Clin Oncol 29: 2773-2780, 2011

2. Gérard JP, Azria D, Gourgou-Bourgade S, Martel-Laffay I, Hennequin C, Etienne PL, Vendrely V, François E, de La Roche G, Bouche $\mathrm{O}$, et al: Comparison of two neoadjuvant chemoradiotherapy regimens for locally advanced rectal cancer: Results of the phase III trial ACCORD 12/0405-Prodige 2. J Clin Oncol 28 $1638-1644,2010$

3. Rödel C, Liersch T, Becker H, Fietkau R, Hohenberger W, Hothorn T, Graeven U, Arnold D, Lang-Welzenbach M, Raab HR, et al: Preoperative chemoradiotherapy and postoperative chemotherapy with fluorouracil and oxaliplatin versus fluorouracil alone in locally advanced rectal cancer: Initial results of the German CAO/ARO/AIO-04 randomised phase 3 trial. Lancet Oncol 13: 679-687, 2012.

4. Park IJ, You YN, Agarwal A, Skibber JM, Rodriguez-Bigas MA, Eng C, Feig BW, Das P, Krishnan S, Crane CH, et al: Neoadjuvant treatment response as an early response indicator for patients with rectal cancer. J Clin Oncol 30: 1770-1776, 2012.

5. Martens MH, Maas M, Heijnen LA, Lambregts DM, Leijtens JW, Stassen LP, Breukink SO, Hoff C, Belgers EJ, Melenhorst J, et al: Long-term outcome of an organ preservation program after neoadjuvant treatment for rectal cancer. J Natl Cancer Inst 108: pii: djw171, 2016.
6. Renehan AG, Malcomson L, Emsley R, Gollins S, Maw A, Myint AS, Rooney PS, Susnerwala S, Blower A, Saunders MP, et al: Watch-and-wait approach versus surgical resection after chemoradiotherapy for patients with rectal cancer (the OnCoRe project): A propensity-score matched cohort analysis. Lancet Oncol 17: 174-183, 2016.

7. Kalady MF, de Campos-Lobato LF, Stocchi L, Geisler DP, Dietz D, Lavery IC and Fazio VW: Predictive factors of pathologic complete response after neoadjuvant chemoradiation for rectal cancer. Ann Surg 250: 582-589, 2009.

8. Russo AL, Ryan DP, Borger DR, Wo JY, Szymonifka J, Liang WY, Kwak EL, Blaszkowsky LS, Clark JW, Allen JN, et al: Mutational and clinical predictors of pathologic complete response in the treatment of locally advanced rectal cancer. J Gastrointest Cancer 45: 34-39, 2014.

9. Zong C, Lu S, Chapman AR and Xie XS: Genome-wide detection of single-nucleotide and copy-number variations of a single human cell. Science 338: 1622-1626, 2012.

10. Armaghany T, Wilson JD, Chu Q and Mills G: Genetic alterations in colorectal cancer. Gastrointest Cancer Res 5: 19-27, 2012.

11. Kaz AM and Brentnall TA: Genetic testing for colon cancer. Nat Clin Pract Gastroenterol Hepatol 3: 670-679, 2006.

12. Martin M: Cutadapt removes adapter sequences from high-throughput sequencing reads. EMBnet J 17: 10-12, 2011.

13. $\mathrm{Li} \mathrm{H}$ and Durbin R: Fast and accurate short read alignment with Burrows-Wheeler transform. Bioinformatics 25: 1754-1760, 2009.

14. Li H, Handsaker B, Wysoker A, Fennell T, Ruan J, Homer N, Marth G, Abecasis G and Durbin R; Genome Project Data Processing S: The sequence alignment/map format and SAMtools. Bioinformatics 25: 2078-2079, 2009.

15. DePristo MA, Banks E, Poplin R, Garimella KV, Maguire JR, Hartl C, Philippakis AA, del Angel G, Rivas MA, Hanna M, et al: A framework for variation discovery and genotyping using next-generation DNA sequencing data. Nature Genet 43: 491-498, 2011.

16. Leung ML, Wang Y, Waters J and Navin NE: SNES: Single nucleus exome sequencing. Genome Biol 16: 55, 2015.

17. Gibson MK, Abraham SC, Wu TT, Burtness B, Heitmiller RF, Heath E and Forastiere A: Epidermal growth factor receptor, p53 mutation and pathological response predict survival in patients with locally advanced esophageal cancer treated with preoperative chemoradiotherapy. Clin Cancer Res 9: 6461-6468, 2003.

18. Villafranca E, Okruzhnov Y, Dominguez MA, García-Foncillas J, Azinovic I, Martinez E, Illarramendi JJ, Arias F, Martinez Monge R, Salgado E, et al: Polymorphisms of the repeated sequences in the enhancer region of the thymidylate synthase gene promoter may predict downstaging after preoperative chemoradiation in rectal cancer. J Clin Oncol 19: 1779-1786, 2001.

19. Hao JJ, Yao HQ, Dai GY, Kang W, Jia XM, Xu X, Cai Y, Zhan QM, Wang GQ and Wang MR: Chromosomal aneuploidies and combinational fluorescence in situ hybridization probe panels are useful for predicting prognosis for esophageal squamous cell carcinoma. J Gastroenterol 50: 155-166, 2015.

20. Li Y, Zhang X, Ge S, Gao J, Gong J, Lu M, Zhang Q, Cao Y, Wang DD, Lin PP, et al: Clinical significance of phenotyping and karyotyping of circulating tumor cells in patients with advanced gastric cancer. Oncotarget 5: 6594-6602, 2014.

21. Gao Y, Zhu Y, Zhang Z, Zhang C, Huang X and Yuan Z: Clinical significance of pancreatic circulating tumor cells using combined negative enrichment and immunostaining-fluorescence in situ hybridization. J Exp Clin Cancer Res 35: 66, 2016.

22. Wu C, Hao H, Li L, Zhou X, Guo Z, Zhang L, Zhang X, Zhong W, Guo H, Bremner RM, et al: Preliminary investigation of the clinical significance of detecting circulating tumor cells enriched from lung cancer patients. J Thorac Oncol 4: 30-36, 2009.

23. Chen Z, Liu Z, Li W, Qu K, Deng X, Varma MG, Fichera A, Pigazzi A and Garcia-Aguilar J: Chromosomal copy number alterations are associated with tumor response to chemoradiation in locally advanced rectal cancer. Genes Chromosomes Cancer 50: 689-699, 2011.

24. Dumont P, Leu JI, Della Pietra AC III, George DL and Murphy M: The codon 72 polymorphic variants of p53 have markedly different apoptotic potential. Nat Genet 33: 357-365, 2003.

25. Johnstone RW, Ruefli AA and Lowe SW: Apoptosis: A link between cancer genetics and chemotherapy. Cell 108: 153-164, 2002.

26. Lin PP: Integrated EpCAM-independent subtraction enrichment and iFISH strategies to detect and classify disseminated and circulating tumors cells. Clin Transl Med 4: 38, 2015. 